\title{
Local DER Driven Grid Support by Coordinated Operation of Devices
}

\author{
C.J. Warmer, M.P.F. Hommelberg, J.K. Kok, I.G. Kamphuis
}

\begin{abstract}
In the traditional operation of electricity networks the system operator has a number of ancillary services available for preservation of system balance. These services are called upon near real-time, after the planning phase.

Ancillary services consist of regulating power, reserve capacity and emergency capacity, each with their own characteristics. Regulating power is deployed via load frequency control. Reserve capacity is used to release regulating power and can be called upon to maintain a balance or to counterbalance or resolve transmission restrictions. Both are traded at the Dutch energy market under an auction model with a single buyer (TenneT). Emergency capacity is rewarded on the basis of accessibility/availability within 15 minutes.

In local electricity networks neither planning nor ancillary services exist. Planning is done by aggregation into large customer groups. For ancillary services one relies on the system operation as sketched above.

In local electricity networks with a large share of distributed generation the costs of keeping the electricity system reliable and stable will increase further and technical problems may arise. The European SmartGrids initiative responds to these challenges in their strategic research agenda. One of the issues addressed in this agenda is the changing role of the distribution grid in which users get a more active role. One opportunity is the introduction of ancillary-type services at the distribution level, utilizing different types of producing and consuming devices in the local network, in order to make the total system more dependable.
\end{abstract}

Distributed generation has a number of characteristics that are similar to characteristics of consumption. Part of it is intermittent / variable, although to a large extent predictable (PV, wind versus lighting, electronic devices). Another part is task-driven (micro-CHP versus electrical heating). Yet another part is controllable or shiftable in time. And storage can behave both ways. The main key words here are flexibility and variability. This flexibility provides a virtual storage capacity within the electricity grid that can be utilized for balancing services at the local grid.

We will present how the PowerMatcher concept, developed by ECN, supports the setting up of local balancing markets in a flexible and logical way. The ICT is already available as an enabling technology. The concept has been demonstrated in several field tests.

Index Terms-- Multi-agent systems, Cooperative systems, Distributed control, Power distribution, Power system control.

C.J. Warmer, M.P.F. Hommelberg, J.K. Kok, I.G. Kamphuis (e-mail \{warmer, hommelberg, j.kok, kamphuis\}@ecn.nl, work in the Intelligent Grids Program at the Energy research Centre of the Netherlands, Westerduinweg 3, P.O. Box 1, 1755 ZG Petten, The Netherlands.

\section{NOMENCLATURE}

CHP - Combined Heat and Power

CRISP- CRitical Infrastructures for Sustainable Power

DER - Distributed Energy Resources

DG - Distributed Generation

DNO - Distribution Network Operator

ECN - Energy research Centre of the Netherlands

ICT - Information and Communication Technology

PRP - Program Responsible Party

PV - Photo-Voltaic

TSO - Transmission System Operator

VPP - Virtual Power Plant

\section{OPERATION OF THE POWER GRID}

O peration of the power delivery infrastructure is a delicate process aimed at obtaining a final balance at real-time between power supply and demand.

\section{A. Phases in grid operation}

Planning phase: Long-term contracts between producers and consumers determine a base load. To adapt to realizations differing from previous estimations and for Program Responsible Parties (PRP) who can decide on a short time range trading is done on a central market, the power exchange. In most countries a program is defined, in which contracted volumes are incorporated. In a program the amount of power generated as a function of time is fixed. Typically a program is defined on a day-ahead basis. As the time of delivery is nearing, slight updates and adjustments of the program are contracted by the program responsible parties in order to adapt to actual power demand. In some countries of Europe an intra-day market is operating for trading surpluses and deficits in capacity and demand.

Ancillary services: In the traditional operation of electricity networks the system operator has a number of ancillary services available for preservation of system balance. These services are called upon near real-time, after the planning phase and are market-based.

Ancillary services consist of regulating power (voltage control, reactive power), different types of reserve capacity and contingency/emergency capacity, each with their own characteristics. Regulating power is deployed via load frequency control. Reserve capacity is used to release regulating power and can be called upon to maintain a balance or to counterbalance or resolve transmission restrictions. Both are traded at the Dutch energy market under an auction model with a single buyer (TenneT). Emergency capacity is rewarded on the basis of 
accessibility/availability within 15 minutes. Depending on the sign of the momentary imbalance, parties supplying or demanding power less than contracted in the planning phase are penalized by the authority.

Local protection: Apart from the above mentioned centralized control systems local protection and control is exerted by components such as circuit breakers and reclosers. Typically this type of control is based on local information only. Novel concepts based on intelligence in the distribution grid by deploying local agents have been developed in the CRISP project [1]. This type of application is not considered in this paper.

\section{B. Distributed energy resources}

Several forces drive a change in the current worldwide energy supply. A main ongoing change is the growing penetration of distributed electricity generation. Volumes of electricity to be traded for which the ancillary market is accessible are beyond the scope of current distributed energy resources. There are two ways to open up the ancillary market for DER. The first one is to aggregate volumes to a level that allows participation on the global ancillary market. This aggregation in so-called virtual power plants is the scope of research in the Fenix project [2]. But large scale penetration of distributed generation may lead to more emphasis on local networks where ancillary services become a necessity. This will create opportunities for DER to deliver ancillary services to distribution networks. To allow DER to perform local tasks and at the same time be active in grid support new control strategies are being developed based on organization of DER using distributed control techniques.

The role of DG in local grid support has also been one of the conclusions from the DG-GRID European project, in which it is stated that "DG through aggregators can participate in balancing and reserve markets. DG can provide voltage support and compensate energy losses as required by DNOs. In the future, with higher levels of network automation and DG controllability, DG would help to solve congestion management, and to improve quality islanding." [3]. The European CRISP project [4] has shown what potential advanced ICT has if used to manage complex networks, evolving through the introduction of large scale DG. Note that in this paper we focus on DER rather than restrict ourselves to DG, including the demand side as well.

\section{POWERMATCHER DISTRIBUTED COORDINATION}

\section{A. The PowerMatcher - a short introduction}

The PowerMatcher is a control concept for coordination of supply and demand in electricity networks with a high share of distributed generation that implements marketbased control theory. It is concerned with optimally using the flexibility of electricity producing and consuming devices to alter their operation in order to increase the overall match between electricity production and consumption.

In the PowerMatcher concept each device is represented by a control agent, which tries to operate the process associated with the device in an economically optimal way. The electricity consumed or produced by the device is bought, respectively sold, by the device agent on an electronic exchange market [5], [6], [7]. The electronic market is implemented in a distributed manner via a network structure in which so-called PowerMatchers, as depicted in Figure 1, coordinate demand and supply of a cluster of devices directly below it. The PowerMatcher in the root of the tree performs the price-forming process; those at intermediate levels aggregate the demand functions of the devices below them. A PowerMatcher cannot tell whether the instances below it are device agents or intermediate PowerMatchers, since the communication interfaces of these are equal. This ensures a standardised interface for all types of devices.

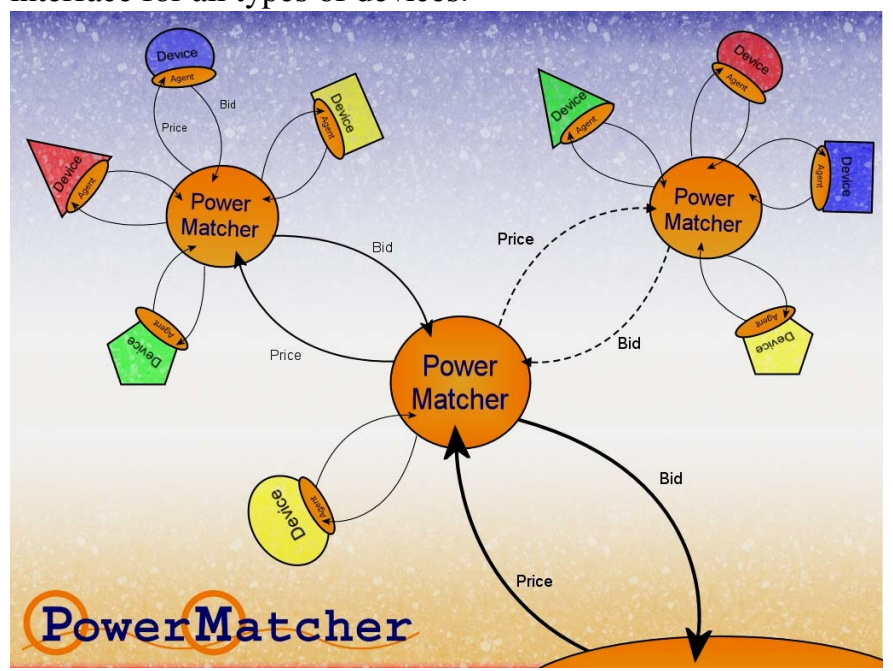

Figure 1 The PowerMatcher architecture; coming from a central tree based mechanism, growing towards a more organic, internet-like structure.

The root PowerMatcher has one or more associated market mechanism definitions, which define the characteristics of the markets, such as the time slot length, the time horizon, and a definition of the execution event (e.g. "every 5 minutes", "every day at twelve o' clock"). When an execution event occurs, the root PowerMatcher sends a request to all directly connected agents to deliver their bids. The device bids are aggregated at the intermediate matchers and passed on up-wards. The root PowerMatcher determines the equilibrium price, which is communicated back to the devices. From the market price and their own bid function each device agent can determine the power allocated to the device.

\section{B. Flexibility of Demand and Supply}

From the viewpoint of controllability, devices that produce or consume electricity fall into a number of main classes, each having a specific agent strategy implemented in the PowerMatcher agent library. An agent strategy is a mapping from the device state history to a demand function shape.

The first class consists of stochastic-operation devices, such as solar and wind energy systems, where the power exchanged with the grid behaves stochastically. As the power output is not controllable, the standard supply function shape is a flat line at a magnitude of the current production level.

The second class is shiftable operation devices, which must run for a certain amount of time regardless of the 
exact moment and thus are shiftable in time. An example of such a device is a ventilation system in a utility building that needs to run for 20 minutes each hour.

The third class comprises thermal buffer devices. Examples of these devices are heating or cooling processes, whose operation objective is to keep a certain temperature within lower and upper limits. Changing standard on/offtype control into price driven control allows for shifting operation to economically attractive moments, while operating limits can still be obeyed. Devices in this category can both be electricity consumers (electrical heating, electrical cooling/freezing) and producers (combined generation of heat and power).

As a fourth class grid-coupled electricity storage devices emerge, such as flywheels, supercapacitators or redox-flow batteries, each with different time-scale of use. They are regarded as an enabling technology for increasing penetration of distributed generation, but currently only are economically feasible in niche applications. Also the expected revolution of the electric vehicle, especially in vehicle-to-grid scenarios will be a driver for the development of grid-coupled storage devices.

Freely controllable devices make up the fifth class. Example is an emergency generator.

A special class form user-activated devices that are expected to be operated on direct user command. From an agent point of view these devices - especially when aggregated into larger clusters - are comparable with stochastic operation devices.

Local agent's self-interested behavior causes electricity consumption to shift toward moments of low electricity prices and causes production to shift toward moments of high prices. So, matching of demand and supply emerges on the global system level.

\section{GRID SUPPORT BY COORDINATION OF DER}

The changing role of the distribution grid and the availability of ubiquitous and low-cost ICT enable a number of potential ways in which distributed energy resources can participate in grid support. Note that we restrict ourselves in the following list to (soft real-time) coordination of devices, and do not take into account stability issues (hard real-time control). Also local protection falls outside the scope of this paper.

Participation in traditional ancillary markets: By aggregation of distributed energy resources into a so-called virtual power plant a (virtual) machine is created that contains the flexibility to deliver regulating services in case of a network imbalance.

Local balancing services: Future electricity networks will have a large degree of local generation to such a degree that local network balancing becomes a major issue. Especially if local networks are capable of running autonomously, such as in the MicroGrids concept, local balancing is has to be contained in the control strategy.

Reactive power compensation: As decentralized generation such as from wind or solar or from $\mu$-CHP in general are devised to supply only real power, reactive power still has to be delivered by the higher grid levels. In a future situation, high-voltage grid parts may temporarily deliver a relatively low amount of real power to their middle-voltage grid parts whilst the reactive power need in these parts remains the same. To adapt to this situation in a flexible way, a significant part of the central generation capacity temporarily may have to be committed to delivering reactive power mainly. As delivering mainly reactive power is not an economically viable way of operating central generation capacity and high-voltage grids, a solution is sought by generating reactive power at the decentralized generators.

Network congestion: If in a distribution system the substation or line capacities are at stake the network operator has to fall down to load shedding techniques in order to alleviate the system. The optimal way to avoid distribution congestion is to avoid peak loads. Coordination if DER devices can become an important tool for network operators if their flexibility of operation is utilized such that demand is shifted to periods with lower demand and supply is shifted to periods with higher demand.

Connection capacity reduction: In critical circumstances network operators may put restrictions on household connection capacity. If not properly guarded this restriction may lead to local outage behind the meter. Local intelligence can prevent this by coordination - at a local level - of household appliances. If distributed generation or storage is available, the household may be able to stay up and running for some time with infringing only low priority task.

\section{PowerMatcher BASEd Field TeStS ANd Simulations}

Although developed as a concept for balancing supply and demand in a cluster of devices, the current version of the PowerMatcher focuses on coordination of supply and demand flows and is capable of providing a variety of services. This is possible by inclusion of so-called business agents that focus on the different goals of these services. In some cases these services can be built around real markets and real prices; in other cases the prices are only meant as a paradigm and it is better to speak of 'value' in stead of 'price'.

Balancing services: Balancing services for network operation closely resembles balancing services at a commercial level. The main difference is that commercial imbalance leads to penalties for the energy supplier, whereas balancing for network operators should lead to a real-time balance. Using the flexibility of distributed devices for balancing therefore are only the first steps in maintaining an instantaneous demand and supply balance in the network.

In [8] the results are described of a field test in the Netherlands aimed at commercial balancing. Wholesale trading parties have to make their production and consumption plan available to the transmission system operator (TSO). We refer to these parties as balancing responsible parties. Deviations from these plans are dealt with by the TSO by contracting capacity for primary, secondary and tertiary control. The cost for these services 
are imposed on the balance responsible parties that are responsible for the deviations. In order to reduce these costs an Imbalance Reduction System been developed, based on the PowerMatcher concept, to assist a PRP in reducing the imbalance of a cluster of devices including wind turbines (see Figure 2). Each 5-15 minutes the production and consumption plan of the cluster is compared with the realtime outcome and the operation of the controllable installations in the cluster (cold store, emergency generator, CHP for district heating and residential heat pumps) is adjusted to minimize the deviation between plan and operation. The operational goals of these devices serve as a constraint. The main focus of the field test is to reduce the wind power dominated imbalance in the cluster.

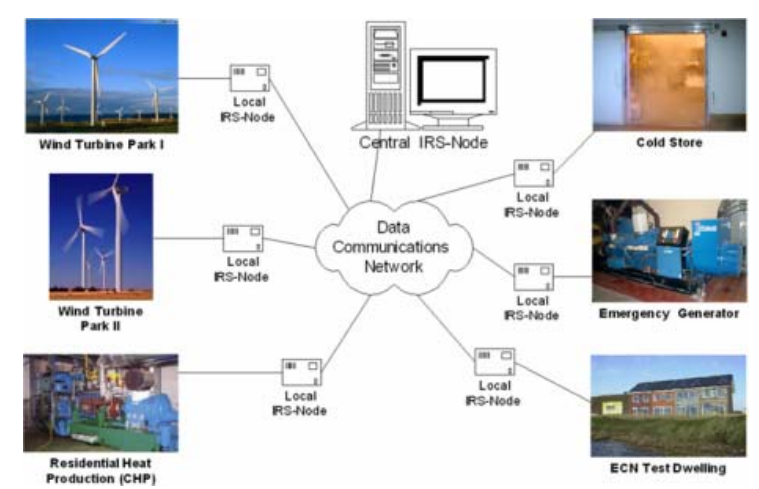

Figure 2 Field test configuration for the Imbalance Reduction System

The total imbalance reduction achieved over an 11-day period is $40 \%$. This reduction has been mainly achieved by compensating for overproduction of wind power. Apparently, there is enough flexibility in the cluster to increase consumption and/or decrease production in these periods. A large part of the underproduction of the wind power has not been compensated. Close analysis of the individual agents' behavior suggest a reason for this. As the weather was quite cold during the measured period, the residential CHP's were scheduled at $100 \%$ operation. This 'must-run' situation did not allow for room to shift production towards the periods of wind underproduction. Yet the CHP's could be partially turned off for some periods without infringing the thermal comfort.

Peak reduction: The First Trial field test in the Netherlands [9] comprises of a cluster of $\mu$-CHP units operated as a virtual power plant, demonstrating their ability to reduce the local peak demand of the common low-voltage grid segment the $\mu$-CHP units are connected to. The field test uses 10 domestic Stirling based $\mu$-CHP units, $1 \mathrm{~kW}_{\mathrm{el}}$ each, at consumer premises.

In this way the VPP supports the local distribution network operator (DNO) to defer reinforcements in the grid infrastructure (substations and cables). Although not all $\mu$ CHP units included in the field test are connected to the same low-voltage cable, during the trial a connection to a common substation (i.e. low-voltage to mid-voltage transformer) is assumed. Note that the field test actually controlled the $\mu$-CHP units at people's homes.

Figure 3 shows the operation for one day in May 2007.
Five $\mu$-CHP units were participating. There is no space heating demand, only demand for tap water heating. The figure shows four demand peaks at the substation, of which the third peak is the least compensated. The second peak takes care of the larger part of the heat demand for tap water. At the third peak, following immediately after the second peak, the heat demand is already largely satisfied. Such a sequence of peaks can no doubt be compensated better during the winter season because of a continuous space heating demand. Simulations have confirmed this expectation. Nevertheless in the situation described a peak reduction of nearly $30 \%$ was obtained.

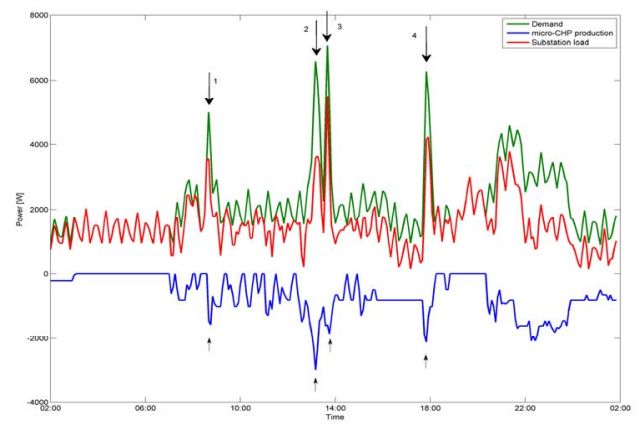

Figure 3 Time series of the substation demand pattern (green), the total $\mu$-CHP production (blue) and the net substation load (red) for $5 \mu$-CHPs with PowerMatcher coordination.

Network congestion: A market outcome such as the PowerMatcher delivers is of no value if it does not yield a network feasible solution. The electricity transport network is subject to congestion by default due to capacity constraints on lines. In [10] a theoretical framework and an algorithmic method are described for finding transport network feasible solutions in market-based flow resource allocation. The algorithm can be built in the PowerMatcher algorithm, thus providing a powerful tool for congestion management in distribution networks.

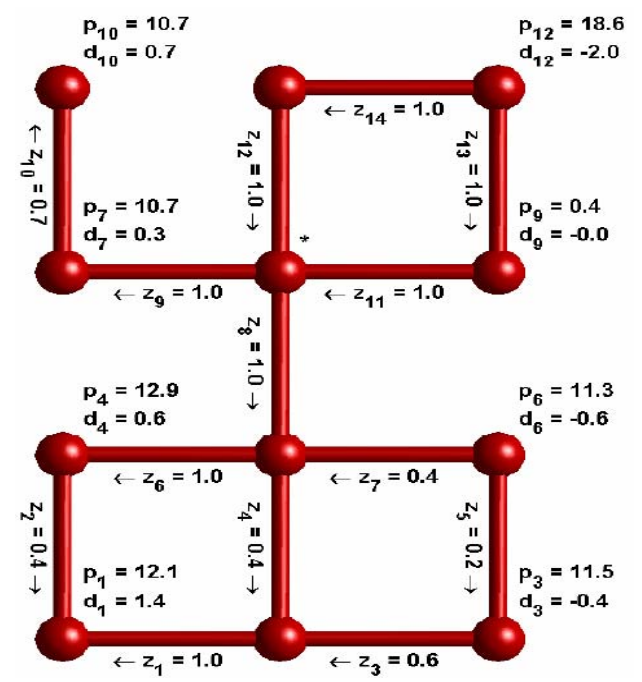

Figure 4 Example network feasible market result. The maximum line capacity is set to 1 for each line. Note that supply is indicated by negative demand.

Figure 4 shows the result of applying the above mentioned algorithm in a network in which the maximum 
capacity of each line has been limited to 1 . The figure shows the flows $\left(\mathrm{z}_{\mathrm{i}}\right)$, the nodal prices $\left(\mathrm{p}_{\mathrm{i}}\right)$ and the corresponding demand/supply $\left(\mathrm{d}_{\mathrm{i}}\right)$.

Note that congestion in a competitive market can lead to price inflation by strategic bidding of generators. These effects have been mentioned by e.g. [11]. Regulating measures have to be taken to tackle this risk. The effect of price inflation is less apparent in networks for which the PowerMatcher is constructed. Not only are many of the generators task bound (e.g. temperature control for CHP units) and therefore less prone to strategic bidding. Also a substantial part of the consumers have the flexibility to alter their demand in case of high prices. Market power is more evenly divided and both sides can apply learning techniques to be better off.

Reactive power compensation: Several approaches have been proposed for local reactive power compensation. In one of these [12] the PowerMatcher approach is taken by letting decentralized generators equipped with power converters bid for reactive power compensation. Simulations of this approach show an effective reduction of the reactive power flow to zero at the sub station. At the moment it is under investigation whether active and reactive power can be traded in one market framework.

\section{CONCLUSION}

The paper describes the potential of the flexibility of distributed energy resources for a number of grid support services. The PowerMatcher concept for market-based coordination of supply and demand is demonstrated in a number of examples, both simulations and field tests. Main advantage of the PowerMatcher above more traditional price-reactive systems is the autonomy with respect to local processes, the scalability with respect to scaling up to large systems with tens of thousands and even millions of nodes. Business models need to be developed.

\section{REFERENCES}

[1] C. Andrieu et al. Fault detection and diagnostics in high-DG distribution systems. Deliverable 1.3 of the CRISP project. 2004.

[2] Fenix has its own website: http://www.fenix-project.org/.

[3] M. Scheepers et al. Regulatory Improvements for Effective Integration of Distributed Generation into Electricity Distribution Networks Summary of the DG-GRID project results. ECN-E-07-083 (2007).

[4] G.J. Schaeffer, J.M. Akkermans. CRISP Final Summary Report. ECNO--07-019, 2007. See also http://crisp.ecn.nl/.

[5] J.K. Kok, C.J. Warmer, I.G. Kamphuis, "PowerMatcher: Multi-agent control in the electricity infrastructure", AAMAS 2005 - 4th International joint conference on Autonomous Agents and MultiAgent Systems, Utrecht, July 2005.

[6] J.K. Kok, C.J. Warmer, I.G. Kamphuis, "The PowerMatcher: Multiagent Control of Electricity Demand and Supply", In M. Pechoucek and S.G. Thompson, "Agents in Industry: The Best from the AAMAS 2005 Industry Track, IEEE Intelligent Systems, March/April 2006.

[7] The PowerMatcher has its own website: http://www.powermatcher.net/.

[8] C.J. Warmer, M.P.F. Hommelberg, I.G. Kamphuis, Z. Derzsi and J.K Kok, "A field test for real-time reduction of intermittent imbalance with the PowerMatcher", Sixth International Workshop on Large-Scale Integration of Wind Power, Delft, 2006.

[9] C.J. Warmer, M. P. F. Hommelberg, B. Roossien, J.K. Kok, J.W. Turkstra - A Field Test Using Agents for Coordination of Residential Micro-CHP. Proceedings of the ISAP Conference, Kaohsiung, 2007.
[10] J.K. Kok \& J.M. Akkermans - Transport Network Feasible Solutions in Market-based Allocation of Flow Resources. in: Salvatore Pennacchio (ed.), Emerging Technologies in Robotics and Control Systems, , June 2007. ISBN: 978-88-901928-9-5.

[11] J. Sun and L. Tesfatsion (2007a), "Dynamic Testing of Wholesale Power Market Designs: An Open-Source Agent-Based Framework", Computational Economics, Volume 30, Number 3, pp. 291-327.

[12] K. Visscher, C.J. Warmer, J.K. Kok. "Local reactive power support for grids with a large share of decentralized generation capacity". CIRED 2007, May 2007.

\section{BIOGRAPHIES}

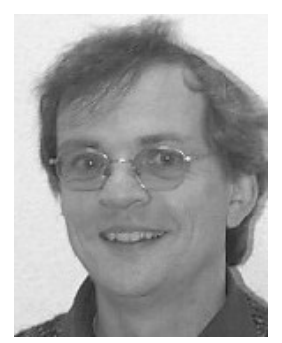

Cor Warmer was born in Utrecht on March 8 1955. He graduated as a mathematical statistician from the Universiteit van Amsterdam in 1981. He joined ECN in 1981 as a mathematics and statistics consultant in a scientific mainframe environment. He was later involved in a large number of projects focusing on data and object modeling. His current research includes process optimization of large energy consuming systems and optimization of power demand and supply flows in the distribution network using market based agent algorithms.

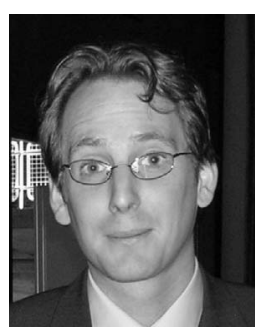

Maarten Hommelberg was born in Tilburg on October 9 1978. He graduated in the field of building services on the University of Technology in Eindhoven in 2005. The graduation subject was titled "Software agents for a building management system". His first and current employer is ECN. He is project leader in the Virtual Power Plant project and is currently working on optimizing power demand and supply flows in the distribution network using market

based agent algorithms.

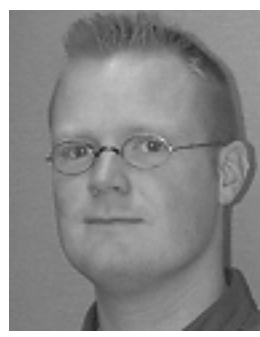

Koen Kok (1969, male) received Bachelor degrees in Electrical Engineering (1992) and Computer Engineering (1992). After a short working period at the University of Groningen, he started to study Computer Science at the same university and received his MSc in Computer Science in 1998. From 1998 to date, he is working as Researcher and Project Co-ordinator at ECN. He is working in the interdisciplinary field between electrical engineering, systems control and ICT. His current research focus is on intelligent distributed control mechanisms for electricity grids with a high penetration of distributed generation.

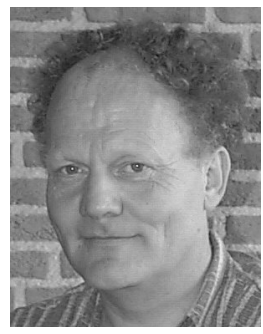

René Kamphuis was born in 1952. He graduated from Nijmegen University in 1976 in Chemistry. After that he got a Ph. D. from Groningen University in Chemical Physics. His employment experience at ECN started with a position at the computing center. From there he went to a number of software engineering positions. Since then he is involved in the Intelligent Energy Grids program at ECN. He has been involved in a number of projects concerning the application of agent technology for comfort management in buildings and dynamic distributed applications in the electricity network. 